\title{
Biomechanical forces promote embryonic haematopoiesis
}

\author{
Luigi Adamo ${ }^{1,{ }^{*},}$, Olaia Naveiras ${ }^{2,},{ }^{*}$ Pamela L. Wenzel ${ }^{2}$, Shannon McKinney-Freeman ${ }^{2}$, Peter \\ J. Mack ${ }^{1}$, Jorge Gracia-Sancho ${ }^{1}$, Astrid Suchy-Dicey ${ }^{1}$, Momoko Yoshimoto ${ }^{3}$, M. William

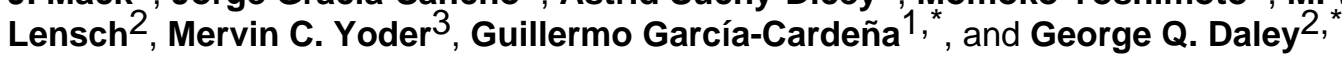 \\ ${ }^{1}$ Center for Excellence in Vascular Biology, Departments of Pathology Brigham and Women's \\ Hospital and Harvard Medical School, Boston, Massachusetts 02115, USA. \\ ${ }^{2}$ Stem Cell Transplantation Program, Division of Pediatric Hematology/Oncology, Children's \\ Hospital Boston and Dana Farber Cancer Institute; Department of Biological Chemistry and \\ Molecular Pharmacology, Harvard Medical School; Division of Hematology, Brigham and Women's \\ Hospital; Harvard Stem Cell Institute; Manton Center for Orphan Disease Research; Howard \\ Hughes Medical Institute, Boston, Massachusetts 02115, USA.
}

${ }^{3}$ Department of Pediatrics, Indiana University School of Medicine, Indianapolis, Indiana 46202, USA.

\begin{abstract}
Biomechanical forces are emerging as critical regulators of embryogenesis, particularly in the developing cardiovascular system ${ }^{1,2}$. After initiation of the heartbeat in vertebrates, cells lining the ventral aspect of the dorsal aorta, the placental vessels, and the umbilical and vitelline arteries initiate expression of the transcription factor Runxl (refs 3-5), a master regulator of haematopoiesis, and give rise to haematopoietic cells ${ }^{4}$. It remains unknown whether the biomechanical forces imposed on the vascular wall at this developmental stage act as a determinant of haematopoietic potential ${ }^{6}$. Here, using mouse embryonic stem cells differentiated in vitro, we show that fluid shear stress increases the expression of Runxl in $\mathrm{CD} 41^{+} \mathrm{c}-\mathrm{Kit}^{+}$haematopoietic progenitor cells ${ }^{7}$,concomitantly augmenting their haematopoietic colony-forming potential. Moreover, we find that shear stress increases haematopoietic colony-forming potential and expression of haematopoietic markers in the paraaortic splanchnopleura/aorta-gonads-mesonephros of mouse embryos and that abrogation of nitric oxide, a mediator of shear-stress-induced signalling ${ }^{8}$, compromises haematopoietic potential in vitro and in vivo. Collectively, these data reveal a critical role for biomechanical forces in haematopoietic development.
\end{abstract}

In the mouse, the first haemogenic areas appear in the yolk sac starting at day 7.5 of development $(\mathrm{E} 7.5)^{9}$. After the establishment of circulation and the onset of vascular flow at day 8.5, additional haemogenic sites appear between day 9 and 10.5 as Runx $1^{+}$regions within

(C)2009 Macmillan Publishers Limited. All rights reserved

Correspondence and requests for materials should be addressed to G.Q.D. (george.daley@ childrens.harvard.edu) or G.G.-C. (guillermo_garcia-cardena@hms.harvard.edu)..

These authors contributed equally to this work.

Author Contributions L.A., O.N, G.G.-C. and G.Q.D. conceived ideas, designed experiments, analysed results and wrote the manuscript. P.J.M. performed the haemodynamic shear stress estimation and programmed the biomechanical stimuli. L.A., O.N., P.L.W., J.G.-S., S.M.-F. and A.S.-D. performed experiments. M.W.L. conceived ideas and contributed to experimental design. M.Y. and M.C.Y. set up timed pregnancies and isolated Ncxl null and wild-type mouse embryos. All authors edited and reviewed the final manuscript. G.Q.D. and G.G.-C. co-directed the project.

Full Methods and any associated references are available in the online version of the paper at www.nature.com/nature.

Supplementary Information is linked to the online version of the paper at www.nature.com/nature.

Reprints and permissions information is available at www.nature.com/reprints. 
the developing vasculature ${ }^{10}$. Given the developmental and anatomical relationship of haematopoietic precursors with the mechano-responsive vascular endothelium ${ }^{11,12}$, together with the temporal correlation between the establishment of circulation and the appearance and expansion of vascular haemogenic sites, we proposed that biomechanical forces might act to promote haematopoiesis.

To explore this hypothesis, we differentiated mouse embryonic stem (ES) cells as embryoid bodies, a system that recapitulates the early stages of embryonic haematopoietic development. In embryoid bodies, cells first commit to mesoderm and then produce Flk $1^{+}$cells containing the earliest embryonic haematopoietic precursors ${ }^{13}$. Embryoid bodies were cultured until the appearance of Flk $1^{+}$mesoderm on day 3.25 of differentiation ${ }^{13,14}$, disaggregated and plated on flat gelatinized surfaces. To define the type of biomechanical stimulation to apply to these cultured cells, we focused on the haemodynamic environment present in the aorta-gonadmesonephros (AGM) region, the best characterized haemogenic site at the onset of circulation ${ }^{15,16}$. The pulsatile characteristics of flow in the aorta generate a complex interplay of distinct types of biomechanical forces including circumferential stress, hydrodynamic pressure and shear stress. Of these forces, we focused on fluid shear stress, the frictional force generated by viscous flow acting along cells lining blood vessels, because it has been shown to exert profound effects on the structure and function of vascular endothelial cells ${ }^{12}$. We therefore chose to stimulate cultured cells using a wall shear stress (WSS) comparable to that acting along the dorsal aorta throughout the cardiac cycle at E10.5. To estimate the timeaverage of this value, we used previously published ultrasound biomicroscopy (UBM) data for the time-average velocity over one cardiac cycle (velocity time integral, $4.74 \mathrm{~mm}$, divided by the cycle length, $350 \mathrm{~ms}$, and the earliest available values for dorsal aorta diameter, $0.33 \mathrm{~mm}$ (refs 10, 17)). Calculating the circular Poiseuille flow solution for wall shear stress with these values yielded a dorsal aorta WSS at E10.5 of approximately $5 \mathrm{dyn} \mathrm{cm}^{-2}$ (Supplementary Fig. 1a). This approach assumed that the UBM velocity data represented mean blood flow and that Poiseuille flow assumptions are valid within the E10.5 dorsal aorta ${ }^{18}$ (see Methods).

Embryoid-body-derived cells were exposed to this biomechanical stimulus (WSS) or cultured under static conditions for $48 \mathrm{~h}$ (Fig. 1a and Supplementary Fig. 1b). Exposure of embryoidbody-derived cells to shear stress caused an increase in cells positive for CD31 (PECAM1), a marker of endothelial ${ }^{19}$ and haematopoietic lineages (Supplementary Fig. 1c). CD $41^{+} \mathrm{c}-\mathrm{Kit}^{+}$ haematopoietic precursors ${ }^{7}$ were sorted by fluorescence-activated cell sorting (FACS). Gene expression analysis within this compartment demonstrated a strong shear-stress-mediated upregulation of the transcription factors Runxl (also called Cbfa2; 4.6-fold) and $M y b$ (2.8fold), the prototypical markers of haemogenic sites ${ }^{4}$, and of Klf2 (4.1-fold), a gene previously shown to be a driver of erythropoiesis ${ }^{20}$ and to be mechano-activated in endothelial cells ${ }^{21}$ (Fig. 1b). Runxl upregulation was observed in the unsorted cell population and this effect was specific to the WSS values estimated for the AGM region in the early embryo. Indeed, when the embryoid-body-derived cells were exposed to a shear stress of different magnitude or to a shear stress waveform characteristic of the human aorta the expression of Runx1 was not increased (Supplementary Fig. 1d).

Next, we interrogated the functional significance of these gene expression changes using haematopoietic colony-forming assays. As a positive control, we used the iCdx4 ES cell line, which carries a doxycycline-inducible $C d x 4$ transgene that enhances differentiation to haematopoietic precursors ${ }^{22}$. Shear stress increased the frequency of haematopoietic colonyforming units (c.f.u.) when compared to static (no shear stress) conditions, with a magnitude comparable to that obtained by induction of $C d x 4$ with doxycycline (Fig. 1c). Notably, the $C d x 4$ transgene was not activated in cells exposed to shear stress (Supplementary Fig. 1e). We observed similar results when we analysed Flk $1^{+}$cells enriched by magnetic cell sorting, indicating that the shear stress response occurs in the Flk1 ${ }^{+}$mesoderm (Supplementary Fig. 
1f). Importantly, the small number of detached cells in static or shear conditions was very similar $(<0.5 \%)$ and the haematopoietic potential in this non-adherent fraction was higher in the cells exposed to WSS (Supplementary Fig. 1g). These observations demonstrate that biomechanical forces enhance embryonic stem-cell-derived haematopoiesis.

To gain mechanistic insights into this process, we investigated the role of nitric oxide (NO), a well characterized signalling pathway strongly regulated by shear stress ${ }^{8}$, and a known modulator of haematopoiesis ${ }^{23}$. Inhibition of NO production via the nitric oxide synthase inhibitor nitro-L-arginine methyl ester (L-NAME) ${ }^{24}$ resulted in a significant reduction of the shear-stress-induced enrichment in c.f.u. as compared with cells treated with the inactive stereoisomer D-NAME (Fig. 2a). Notably, L-NAME did not affect shear-stress-induced Runxl upregulation in $\mathrm{CD} 41^{+} \mathrm{c}-\mathrm{Kit}+$ cells, indicating that shear-stress-mediated Runx 1 upregulation is upstream of NO production (Fig. 2b). L-NAME and D-NAME had no effect on c.f.u. formation or Runxl expression in cells grown under static conditions (Supplementary Fig. 2a, b).

We next sought to determine the role of NO production on intraembryonic haematopoiesis in vivo. We administered L-NAME or D-NAME to pregnant mice for $48 \mathrm{~h}$, starting from the time of establishment of circulation (E8.5), and assessed the number of c.f.u. in the AGM regions of E10.5 embryos. As shown in Fig. 2c, systemic inhibition of NO production led to a marked decrease in the number of c.f.u. per AGM. In this in vivo setting, we cannot distinguish between the direct effect of NO inhibition on haematopoietic precursors and the alterations of embryonic haemodynamics that result from changes in vascular tone triggered by the systemic inhibition of NO synthases ${ }^{24}$. However, our in vitro data document that NO production is required for the shear-stress-mediated stimulation of haematopoietic progenitors, thereby establishing that the NO pathway is an important mediator of the effect of shear stressin haematopoiesis.

To examine the effect of fluid shear stress on the haematopoietic forming potential of embryonic haematopoietic sites, we established two-dimensional primary cultures of paraaortic splanchnopleura (PSp, the precursor of the AGM in E9-E9.5 embryos) or AGM-derived cells. When PSp regions from E9.5 murine embryos were disaggregated, plated and exposed to shear stress, we observed an increase in c.f.u. when compared to static controls (Fig. 3a). Likewise, when we exposed cells isolated from the AGM regions of E10.5 murine embryos to shear stress, we observed enhanced expression of Runx1 and Klf2, but for unclear reasons not Myb, in $\mathrm{CD}_{4} 1^{+}$haematopoietic progenitors enriched by FACS (Fig. 3b) ${ }^{25}$. Shear stress also induced upregulation of CD31 ${ }^{+}$cells (Fig. 3c). We then examined the effect of shear stress on specific haematopoietic lineages by flow cytometry, and documented an increase in cells positive for the B lymphocyte marker B220, and Ter119, a marker of erythroblasts (Fig. 3d and Supplementary Fig. 3a). Furthermore, the erythroblasts present in the shear-stress-treated cultures displayed morphological features consistent with later stages of erythroid maturation (that is, pycnotic erythroblasts and a haemoglobinized red pellet versus earlier polychromatic erythro-blasts present in the static control; Fig. 3d and Supplementary Fig. 3b), suggesting accelerated erythroid differentiation in response to shear stress. This was associated with strong upregulation of $K l f 2$, a known driver of erythropoiesis. Taken together, these observations show that shear stress increases both the prevalence of haematopoietic progenitors and the expression of haematopoietic markers in primary cultures of cells taken from the PSp/AGM, indicating that shear stress acts to enhance embryonic haematopoiesis.

To evaluate further the relevance of shear stress in the maturation of embryonic haemogenic sites in vivo, we assessed the effect of fluid shear stress in mouse embryos carrying a homozygous mutation in the $\mathrm{Na}^{+} / \mathrm{Ca}^{2+}$ exchanger $N c x l$ (also called Slc8al). These embryos fail to initiate the heartbeat and therefore lack circulation ${ }^{26} . N c x 1^{-/-}$embryos are deficient in haematopoietic c.f.u. in the PSp, possibly due to a failure of redistribution from the yolk 
$\mathrm{sac}^{27}$, and have a 6-fold reduction in $\mathrm{CD} 41^{+}$haematopoietic progenitors in the placental vasculature ${ }^{5}$. The data presented here suggest that the haematopoietic deficiencies observed in $\mathrm{Ncxl}^{-/-}$embryos may be due, at least in part, to a lack of biomechanical stimulation at haemogenic vascular sites. To evaluate this possibility, we determined the levels of Runxl and Klf2 expression in the PSp/AGM region of E9.25 $\mathrm{Ncxl}^{-1-}$ embryos and wild-type littermates. As shown in Fig. 4a, both Runxl and $K l f 2$ were expressed in $\mathrm{Ncxl}^{-1-}$ PSp/AGM region at significantly lower levels than in controls, whereas expression of the endothelial-specific marker VE-cadherin was similar in the two groups, suggesting that the content of vascular endothelial tissue was similar in both samples (data not shown). Importantly, at this developmental stage, Runxl is exclusively expressed in haemogenic sites within the embryo trunk $^{28,29}$. We then examined if exposure to biomechanical stimulation per se could restore expression of Runx 1 and haematopoietic colony-forming potential in $\mathrm{Ncxl}^{-/-}$embryo-derived cells. We isolated cells from the PSp/AGM region of $\mathrm{Ncx}^{-1-}$ embryos, and either maintained them under static conditions or exposed them to shear stress. As shown in Fig. 4, shear stress induced Runx 1 expression (Fig. 4b) and c.f.u. activity (Fig. 4c) in cells from $\mathrm{Ncx}^{-1-}$ embryos. These data demonstrate that shear stress is capable of upregulating expression of the haematopoietic master regulator Runxl and promoting haematopoietic colony-forming activity in cells derived from the PSp region of $\mathrm{Ncxl}^{-/-}$embryos, and indicates that the haematopoietic deficiency of $\mathrm{Ncx}^{-/-}$embryos is not a cell-autonomous defect but rather a consequence of alterations in haemodynamics affecting vascular haemogenic sites.

Our results demonstrate that biomechanical forces stimulate embryonic haematopoiesis both in ES cell cultures and within murine embryos. These data establish a link between the initiation of the heartbeat, vascular flow and embryonic haematopoietic development, and provide new perspectives for the manipulation and production of haematopoietic progenitors from pluripotent stem cells in vitro, a key process for the implementation of stem-cell-based therapy of haematological diseases.

\section{METHODS SUMMARY}

Haemodynamic shear stress in the embryonic aorta was estimated using previously published fluid dynamic data from the developing mouse embryo ${ }^{10,17,30}$ and assuming circular Poiseuille flow. Ainv15 CDX4-inducible mouse embryonic stem cells (iCDX4 ES) were cultured as described $^{22}$ and differentiated via the hanging-drop embryoid body method. Differentiated ES cells were plated at 100,000 per $\mathrm{cm}^{2}$ on $95 \mathrm{~cm}^{2}$ plates, and exposed to shear stress in the presence or absence of $2 \mathrm{mM} \mathrm{N}(\mathrm{G})$-nitro-L-arginine methyl ester (L-NAME) or $2 \mathrm{mM} \mathrm{N}(\mathrm{G})$ nitro-D-arginine methyl ester (D-NAME). Pregnant Swiss-Webster mice were purchased from Taconic farms. Noon on the day the copulation plug was found was designated 0.5 days of gestation (E0.5). PSp/AGM regions were dissected with a conservative approach, preserving the somites. Two-dimensional primary PSp/AGM cultures were optimized in the absence or presence of growth factors (ECGS, heparin, SCF, VEGF, TPO and Flt3 ligand).

\section{METHODS}

\section{Mouse ES cell culture and differentiation}

Ainv15 CDX4-inducible mouse embryonic stem cells (iCDX4 ES) were cultured on mitomycin-treated mouse embryonic fibroblasts (MEFs) (Chemicon, strain CF-1) and passaged every $2-3$ days with $0.25 \%$ trypsin/EDTA as previously described ${ }^{22}$. To induce differentiation, ES cells were trypsinized, gently dispersed by repetitive pipetting and replated for $45 \mathrm{~min}$ at $37^{\circ} \mathrm{C}$ for MEF depletion. Floating and loosely adherent cells were gathered, checked for viability with trypan blue, and diluted in embryoid body differentiation media (IMDM, 10\% FCS Stem Cell technologies catalogue number 06952, 10 units $\mathrm{ml}^{-1}$ penicillin,

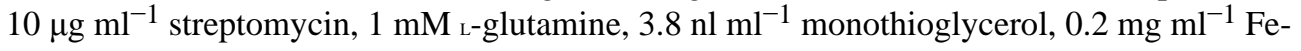


saturated transfer-rin, $0.5 \mathrm{mg} \mathrm{ml}^{-1}$ ascorbic acid) to a final concentration of $6.7 \times 10^{3}$ cell $\mathrm{ml}^{-1}$. Embryoid bodies were cultured via the hanging-drop method in $15-\mu l$ drops on $15 \mathrm{~cm}$ bacterial dishes. Typically $10015-\mathrm{cm}$ plates of embryoid bodies were used per experiment. After $48 \mathrm{~h}$, embryoid bodies were pooled into calcium- and magnesium-containing warm PBS and collected by sedimentation in 50-ml Falcon tubes. Pooled embryoid bodies were transferred to $10-\mathrm{cm}$ tissue culture dishes containing $10 \mathrm{ml}$ of embryoid body differentiation media each, placed on an orbital shaker moving at 75 r.p.m., and incubated for an additional $30 \mathrm{~h}$. After this second culture phase, day 3.25 embryoid bodies were collected by precipitation, washed two times in 30-ml calcium-free PBS and trypsinized for $1 \mathrm{~min}$ in a 1:1 dilution of calcium-free PBS and $0.25 \%$ Trypsin-EDTA. Trypsinization was blocked by addition of 10 $\mathrm{ml}$ embryoid body differentiation media.

\section{Haemodynamic shear-stress estimation}

Wall shear stress acting along the dorsal aorta transiently increases during embryonic development once the heart begins beating. This increase in shear stress may be attributed to both an increase in blood flow velocity, a result of increasing cardiac output ${ }^{31,32}$, and an increase in viscosity, an effect of increasing haematocrit ${ }^{33}$. However, during the first two embryonic days after the initiation of a beating heart (E8-E10), the haematocrit rises, but remains below $20 \%$. This change has an insignificant contribution to blood viscosity ${ }^{34}$ and, therefore, at these early time points blood viscosity can be assumed constant and equal to 0.015 dyn s$~^{-1} \mathrm{~cm}^{-2}$. Using ultrasound biomicroscopy (UBM) data, one study ${ }^{32}$ has previously determined blood flow in the developing embryo dorsal aorta to be laminar throughout the cardiac cycle with minimal skewing of the spatial velocity profile, as determined by calculation of non-dimensional fluid dynamic parameters at E11.5, including the Reynolds number (157), Womersley parameter (0.63) and Dean number (39), respectively. Assuming such fluid dynamic parameters remain valid for earlier time points during development, as well as that the instantaneous blood velocity profile is parabolic, we estimated the dorsal aorta wall shear stress based on circular Poiseuille flow in which the wall shear stress, $\tau$, is calculated by $\tau=$ $8 \mu V / D$, where $\mu$ is the apparent viscosity $\left(\right.$ dyn s$\left.^{-1} \mathrm{~cm}^{-2}\right), V\left(\mathrm{~mm} \mathrm{~s}^{-1}\right)$ is the time-average mean blood flow velocity, and $D(\mathrm{~mm})$ is the vessel diameter. A similar strategy ${ }^{33}$ has been used to estimate embryonic shear stress levels in the developing embryo (E8.5-10.5).

\section{Mouse ES cell exposure to shear stress}

Differentiated ES cells were plated at 100,000 per $\mathrm{cm}^{2}$ on horizontal $95-\mathrm{cm}^{2}$ plates, compatible with a Dynamic Flow System ${ }^{35}$. The plates were coated with a thick layer of $1 \%$ gelatin (DIFCO 214340, Becton Dickinson). After $12 \mathrm{~h}$, cells were washed three times with $10 \mathrm{ml}$ of warm PBS with calcium and incubated for 45 min with embryoid body differentiation media with or without $2 \mathrm{mM} \mathrm{N}(\mathrm{G})$-nitro-L-arginine methyl ester (L-NAME) or $2 \mathrm{mM} \mathrm{N}(\mathrm{G})$-nitro-darginine methyl ester (D-NAME) (Sigma-Aldrich). Following 45 min of incubation time, cells were either moved to the Dynamic Flow System and exposed to shear stress or maintained in a standard cell culture incubator. The shear stress ramped in a step-wise fashion from 0 to 5 dyn $\mathrm{cm}^{-2}$ over a period of $10 \mathrm{~h}$. Following the 10 -h ramping period, the cells were then exposed to a constant $5 \mathrm{dyn} \mathrm{cm} \mathrm{cm}^{-2}$ shear stress for $38 \mathrm{~h}$. In cells exposed to shear stress, culture media with or without D-NAME or L-NAME was automatically exchanged and stored at $37^{\circ} \mathrm{C}$ and $5 \% \mathrm{CO}_{2}$. In static controls, culture media was replaced after $24 \mathrm{~h}$ and stored in $75-\mathrm{cm}^{2}$ flasks in a cell culture incubator. After $48 \mathrm{~h}$ exposure to shear stress or growth under static conditions, cells were detached by 10 min incubation with a 1:1 dilution of Trypsin-Versene mixture (Cambrex, number 17-161E) and Hank's Balanced Salt Solution. 


\section{Primary AGM/PSp culture}

E9.25/9.5 or E10.5 murine embryos were collected in PBS, and a conservative dissection of the AGM/PSp region preserving the somites was performed. Typically, 32 embryos were used per condition on either the full Dynamic Flow System cell growth surface area $\left(95 \mathrm{~cm}^{2}, \mathrm{E} 10.5\right.$ embryos) or about one-third of the Dynamic Flow System cell growth surface area $\left(38 \mathrm{~cm}^{2}\right.$, E9.5). For the $\mathrm{Ncxl}^{-1-}$ rescue experiment, single E9.25 PSp were disaggregated independently, divided in two and plated at a density of half embryo equivalent per $0.3 \mathrm{~cm}^{2}$ either in the Dynamic Flow System or in a 96-well format system. E9.5/E9.25 embryos were dissected within a maximum of $2.5 \mathrm{~h}$ and exposed to dispase (see below for details) without any delay. PSp-derived cells were plated in M5300 Myelocult medium (Stem Cell technologies) in the absence of hydrocortisone on surfaces coated for $1 \mathrm{~h}$ with a 1:20 matrigel dilution (Beckton Dickinson), cells adhered for $6.5 \mathrm{~h}$ and were then moved to the shear stress culture system for $36 \mathrm{~h}$ in either static condition or exposed to shear stress. When culturing cells in 96-well format, Myelocult was enriched with $25 \mathrm{ml} \mathrm{l}^{-1}$ of 1 M HEPES (Invitrogen). The shear stress ramped in a step-wise fashion from 0 to $5 \mathrm{dyn} \mathrm{cm}^{-2}$ over a period of $10 \mathrm{~h}$. After the 10-h ramping period, as described in Supplementary Fig. 1a, the cells were exposed to a constant 5 dyn $\mathrm{cm}^{-2}$ shear stress for $26 \mathrm{~h}$. Cells were harvested by dissociation with $0.1 \%$ dispase for $20 \mathrm{~min}$. E10.5 AGM regions were dissociated in dispase in the same manner. E10.5 AGM-derived cells were plated overnight on $1 \%$ gelatin-coated surfaces in IMDM 10\% FCS, 1× NEAA (Gibco),

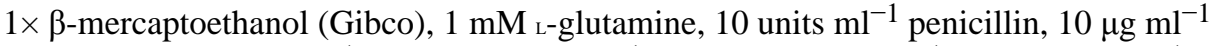
streptomycin, $50 \mu \mathrm{g} \mathrm{ml}^{-1}$ ECGS, $100 \mu \mathrm{g} \mathrm{ml}^{-1}$ heparin, $100 \mathrm{ng} \mathrm{ml}^{-1} \mathrm{SCF}, 40 \mathrm{ng} \mathrm{ml}^{-1}$ VEGF, $40 \mathrm{ng} \mathrm{ml}^{-1} \mathrm{TPO}$ and $100 \mathrm{ng} \mathrm{ml}^{-1} \mathrm{Flt} 3$ ligand overnight. IMDM media supplementation was then reduced to $25 \mu \mathrm{g} \mathrm{ml}^{-1}$ ECGS, $50 \mu \mathrm{g} \mathrm{ml}^{-1}$ heparin, $25 \mathrm{ng} \mathrm{ml}^{-1} \mathrm{SCF}, 20 \mathrm{ng} \mathrm{ml}^{-1}$ VEGF, $10 \mathrm{ng} \mathrm{ml}^{-1}$ TPO and $25 \mathrm{ng} \mathrm{ml}^{-1}$ Flt3 ligand when cells were moved to the Dynamic Flow System and kept for $48 \mathrm{~h}$ in either static condition or exposed to shear stress. The shear stress ramped in a step-wise fashion from 0 to $5 \mathrm{dyn} \mathrm{cm}^{-2}$ over a period of $10 \mathrm{~h}$. After the $10-\mathrm{h}$ ramping period, the cells were then exposed to a constant $5 \mathrm{dyn} \mathrm{cm}^{-2}$ shear stress for $38 \mathrm{~h}$. Cells were harvested as described herein for mouse ES-derived cells. Non-adherent cells were gathered by centrifugation. Embryos derived from $\mathrm{NcxI}^{+/-}$mothers were identified as mutant $\left(\mathrm{Ncx}^{-1-}\right)$ or heterozygous/wild type by visual inspection of the yolk sac vasculature. Genotype was further confirmed by automated genotyping (Transnetyx) on embryonic biopsies collected at the time of dissection. Dispase stock solution was prepared diluting the commercially available powder form (GIBCO) into PBS without calcium or magnesium to a final concentration of $2.5 \mathrm{~g}$ per $100 \mathrm{ml}$. The solution was filtered in $0.22 \mu \mathrm{M}$ tissue culture filters and stored at $-20^{\circ} \mathrm{C}$. The stock was diluted 1:20 in PBS with calcium and magnesium to make working solutions. Embryonic tissue was disaggregated by incubating it in dispase working solution for $40 \mathrm{~min}$ in a thermal mixer rotating a 1,100 r.p.m. at $37^{\circ} \mathrm{C}$. After the first $20 \mathrm{~min}$ of incubation, dispase solution was pipetted up and down about 15 times to break larger tissue pieces. To detach cells plated on matrigel, cells were covered in dispase working solution, incubated $20 \mathrm{~min}$ in a $37^{\circ} \mathrm{C}$ incubator and then collected by pipetting. Before removing dispase, cells were incubated for another $20 \mathrm{~min}$ in a $37^{\circ} \mathrm{C}$ water bath.

\section{RNA extraction and quantitative real-time PCR}

Total RNA was extracted with the QIAGEN RNAeasy Micro Kit, according to the manufacturer's instructions. For RNA extraction from $\mathrm{CD} 41^{+} \mathrm{c}-\mathrm{Kit}^{+}$cells, approximately 7,000 cells were sorted directly into $300 \mu \mathrm{l}$ of lysis buffer. Reverse transcription of RNA was performed using Applied Biosystems Multiscribe DNA polymerase, according to the manufacturer's instructions. When processing FACS-sorted cells, RNA was eluted in $20 \mu \mathrm{l}$ and all collected RNA was used in $50 \mu \mathrm{l}$ reverse transcription reactions. Real-time Taqman PCR (Applied Biosystems) was performed in $20 \mu \mathrm{l}$ reactions with primers provided by Applied Biosystems, according to the manufacturer's instructions. 


\section{FACS sorting and analysis}

Cells were incubated with Fc Block antibody (BD 553141, dilution 1:100) and then stained with anti-CD41 antibody (BD 553848, 1:150 dilution), anti-c-Kit antibody (BD 553356, 1:150 dilution), anti-Flk1 antibody (BD 555308, 1:150 dilution), anti-CD31 antibody (BD 553372, 1:150 dilution) and 7AAD (BD 559925, 1:75 dilution) and sorted with a FACS-Aria system (BD Biosciences).

\section{Methylcellulose c.f.u. assay}

Methylcellulose assay was performed as previously described ${ }^{36}$ in M3434 complete methylcellulose (Stem Cell Technologies) and scored on day 8-10 after plating. When assessing c.f.u. activity on cells exposed to shear stress in 96-well format $\left(\mathrm{Nc}_{\mathrm{C}} \mathrm{I}^{-{ }^{--}} \mathrm{PSp}\right.$-derived cells), at the end of the experiment shear stress media was exchanged with $100 \mu 1$ of M3434 complete methylcellulose medium. Cells were allowed to grow in M3434 in the 96-well plate for 4 days and then replated in $2 \mathrm{ml}$ of complete M3434 media in optical quality 35-mm dishes. To replate, $100 \mathrm{ml}$ of IMDM 15\% FCS was added to the wells and cells were collected by repeated pipetting before washing the well with additional $100 \mu \mathrm{l}$ of IMDM 15\% FCS. Plates were scored on day 14 after replating.

\section{Blockade of NO production in vivo}

Day 7 post coitum timed-pregnant Swiss-Webster mice were purchased from Taconic Farms. Noon on the day the copulation plug was found was designated 0.5 days. On day E8.5 (day 9 post coitum) mice were intraperitoneally injected with $300 \mathrm{mg} \mathrm{kg}^{-1}$ of L-NAME or D-NAME (Sigma) dissolved in sterile, apyrogenic saline solution using a dose volume of $10 \mathrm{ml} \mathrm{kg}^{-1}$ (ref. ${ }^{37}$ ). The injection was repeated after $24 \mathrm{~h}$. Exactly $24 \mathrm{~h}$ after the second injection, mice were killed with $\mathrm{CO}_{2}$, embryos were harvested, and a conservative dissection of the AGM was performed. Embryo trunks were dissociated with $0.1 \%$ dispase and analysed with the methylcellulose assay. Experiments were carried out with Institutional Animal Care and Use Committee approval from Harvard Medical School or Children's Hospital, Boston.

\section{Statistical analysis}

All the statistical analyses were performed using un-paired two-tailed Student's $t$-test assuming experimental samples of equal variance, unless otherwise specified.

\section{Supplementary Material}

Refer to Web version on PubMed Central for supplementary material.

\section{Acknowledgments}

We thank G. Losyev for assistance with flow cytometry, S. Schmitt for critical help in optimizing AGM culture conditions, C. Lengerke and Y. Mukouyama for critical discussions. L.A. was partially funded by the Giovanni Armenise-Harvard Foundation. O.N. was partially funded by the Barrie de la Maza Foundation. G.G.-C. was supported by grants from the National Institutes of Health and G.Q.D was supported by grants from the National Institutes of Health (NIH), and the NIH Director's Pioneer Award of the NIH Roadmap for Medical Research. G.Q.D. is a recipient of the Burroughs Wellcome Fund Clinical Scientist Award in Translational Research and is an Investigator of the Howard Hughes Medical Institute.

\section{References}

1. Hove JR, et al. Intracardiac fluid forces are an essential epigenetic factor for embryonic cardiogenesis. Nature 2003;421:172-177. [PubMed: 12520305]

2. Lucitti JL, et al. Vascular remodeling of the mouse yolk sac requires hemodynamic force. Development 2007;134:3317-3326. [PubMed: 17720695] 
3. Garcia-Porrero JA, Godin IE, Dieterlen-Lievre F. Potential intraembryonic hemogenic sites at preliver stages in the mouse. Anat. Embryol 1995;192:425-435. [PubMed: 8546334]

4. North TE, et al. Runx1 expression marks long-term repopulating hematopoietic stem cells in the midgestation mouse embryo. Immunity 2002;16:661-672. [PubMed: 12049718]

5. Rhodes KE, et al. The emergence of hematopoietic stem cells is initiated in the placental vasculature in the absence of circulation. Cell Stem Cell 2008;2:252-263. [PubMed: 18371450]

6. Lensch MW, Daley GQ. Origins of mammalian hematopoiesis: in vivo paradigms and in vitro models. Curr. Top. Dev. Biol 2004;60:127-196. [PubMed: 15094298]

7. Mikkola HK, Fujiwara Y, Schlaeger TM, Traver D, Orkin SH. Expression of CD41 marks the initiation of definitive hematopoiesis in the mouse embryo. Blood 2003;101:508-516. [PubMed: 12393529]

8. Garcia-Cardena G, et al. Dynamic activation of endothelial nitric oxide synthase by Hsp90. Nature 1998;392:821-824. [PubMed: 9580552]

9. Haar JL, Ackerman GA. A phase and electron microscopic study of vasculogenesis and erythropoiesis in the yolk sac of the mouse. Anat. Rec 1971;170:199-223. [PubMed: 4931266]

10. Ji RP, et al. Onset of cardiac function during early mouse embryogenesis coincides with entry of primitive erythroblasts into the embryo proper. Circ. Res 2003;92:133-135. [PubMed: 12574139]

11. Tavian M, et al. The vascular wall as a source of stem cells. Ann. NY Acad. Sci 2005;1044:41-50. [PubMed: 15958696]

12. Garin G, Berk BC. Flow-mediated signaling modulates endothelial cell phenotype. Endothelium 2006;13:375-384. [PubMed: 17169770]

13. Kabrun N, et al. Flk-1 expression defines a population of early embryonic hematopoietic precursors. Development 1997;124:2039-2048. [PubMed: 9169850]

14. Lengerke C, et al. BMP and WNT specify hematopoietic fate by activation of the CDX-Hox pathway. Cell Stem Cell 2008;2:72-82. [PubMed: 18371423]

15. Medvinsky A, Dzierzak E. Definitive hematopoiesis is autonomously initiated by the AGM region. Cell 1996;86:897-906. [PubMed: 8808625]

16. Cumano A, Dieterlen-Lievre F, Godin I. Lymphoid potential, probed before circulation in mouse, is restricted to caudal intraembryonic splanchnopleura. Cell 1996;86:907-916. [PubMed: 8808626]

17. Phoon CK, Aristizabal O, Turnbull DH. Spatial velocity profile in mouse embryonic aorta and Doppler-derived volumetric flow: a preliminary model. Am. J. Physiol. Heart Circ. Physiol 2002;283:H908-H916. [PubMed: 12181118]

18. Ku D. Blood flow in arteries. Annu. Rev. Fluid Mech 1997;29:399-434.

19. Yamamoto K, et al. Fluid shear stress induces differentiation of Flk-1-positive embryonic stem cells into vascular endothelial cells in vitro. Am. J. Physiol. Heart Circ. Physiol 2005;288:H1915-H1924. [PubMed: 15576436]

20. Basu P, et al. KLF2 is essential for primitive erythropoiesis and regulates the human and murine embryonic beta-like globin genes in vivo. Blood 2005;106:2566-2571. [PubMed: 15947087]

21. Parmar KM, et al. Integration of flow-dependent endothelial phenotypes by Kruppel-like factor 2. J. Clin. Invest 2006;116:49-58. [PubMed: 16341264]

22. Wang Y, Yates F, Naveiras O, Ernst P, Daley GQ. Embryonic stem cell-derived hematopoietic stem cells. Proc. Natl Acad. Sci. USA 2005;102:19081-19086. [PubMed: 16357205]

23. Aicher A, et al. Essential role of endothelial nitric oxide synthase for mobilization of stem and progenitor cells. Nature Med 2003;9:1370-1376. [PubMed: 14556003]

24. Rees DD, Palmer RM, Moncada S. Role of endothelium-derived nitric oxide in the regulation of blood pressure. Proc. Natl Acad. Sci. USA 1989;86:3375-3378. [PubMed: 2497467]

25. Ferkowicz MJ, et al. CD41 expression defines the onset of primitive and definitive hematopoiesis in the murine embryo. Development 2003;130:4393-4403. [PubMed: 12900455]

26. Koushik SV, et al. Targeted inactivation of the sodium-calcium exchanger (Ncx1) results in the lack of a heartbeat and abnormal myofibrillar organization. FASEB J 2001;15:1209-1211. [PubMed: 11344090]

27. Lux CT, et al. All primitive and definitive hematopoietic progenitor cells emerging prior to E10 in the mouse embryo are products of the yolk sac. Blood 2007;111:3435-3438. [PubMed: 17932251] 
28. Simeone A, Daga A, Calabi F. Expression of runt in the mouse embryo. Dev. Dyn 1995;203:61-70. [PubMed: 7647375]

29. North T, et al. Cbfa2 is required for the formation of intra-aortic hematopoietic clusters. Development 1999;126:2563-2575. [PubMed: 10226014]

30. Jones EA, Baron MH, Fraser SE, Dickinson ME. Measuring hemodynamic changes during mammalian development. Am. J. Physiol. Heart Circ. Physiol 2004;287:H1561-H1569. [PubMed: 15155254]

31. Ji RP, et al. Onset of cardiac function during early mouse embryogenesis coincides with entry of primitive erythroblasts into the embryo proper. Circ. Res 2003;92:133-135. [PubMed: 12574139]

32. Phoon CK, Aristizabal O, Turnbull DH. Spatial velocity profile in mouse embryonic aorta and Doppler-derived volumetric flow: a preliminary model. Am. J. Physiol. Heart Circ. Physiol 2002;283:H908-H916. [PubMed: 12181118]

33. Jones EA, Baron MH, Fraser SE, Dickinson ME. Measuring hemodynamic changes during mammalian development. Am. J. Physiol. Heart Circ. Physiol 2004;287:H1561-H1569. [PubMed: 15155254]

34. Nosek, TM. Essentials of Human physiology — Cardiac and Circulatory Physiology. Gold Standard Multimedia; 2000.

35. Blackman BR, Garcia-Cardena G, Gimbrone MA Jr. A new in vitro model to evaluate differential responses of endothelial cells to simulated arterial shear stress waveforms. J. Biomech. Eng 2002;124:397-407. [PubMed: 12188206]

36. Wang Y, Yates F, Naveiras O, Ernst P, Daley GQ. Embryonic stemcell-derived hematopoietic stem cells. Proc. Natl Acad. Sci. USA 2005;102:19081-19086. [PubMed: 16357205]

37. Tiboni GM, Marotta F, Barbacane L. Production of axial skeletalmalformations with the nitric oxide synthesis inhibitorNG-nitro-l-argininemethyl ester (L-NAME) in the mouse. Birth Defects Res. B Dev. Reprod. Toxicol 2007;80:28-33. [PubMed: 17294455] 
a
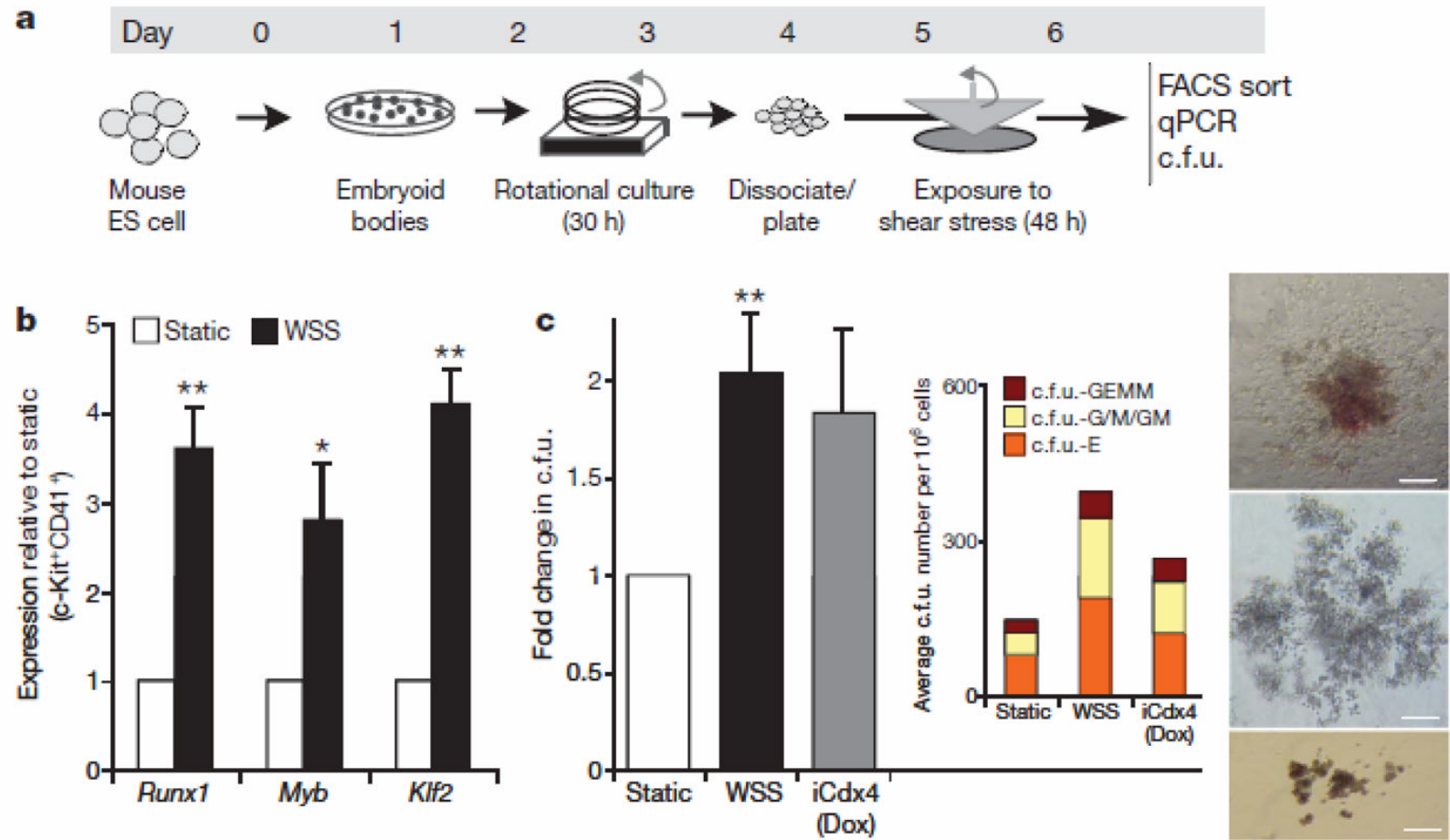

Figure 1. Shear stress induces haematopoietic commitment from ES-derived cells

a, Experimental protocol used to induce haematopoietic differentiation from ES-derived cells in the presence of wall shear stress (WSS). ES cells are differentiated with the embryoid body method for 3.25 days, disaggregated and plated on gelatinized surfaces. Cell monolayers were exposed to shear stress and then collected on day 6 for further analysis b, Real-time Taqman PCR-based gene expression analysis in FACS-sorted CD $41^{+} \mathrm{c}-\mathrm{Kit}^{+}$embryoid-body-derived haematopoietic precursors. Exposure to WSS induces upregulation of the haematopoietic markers Runxl $(P=0.01), M y b(P=0.03)$ and Klf2 $(P=0.001), n=3$. c, Methylcellulose haematopoietic c.f.u. assay. WSS increases the frequency of haematopoietic progenitors in complete M3434 methylcellulose. $n=4$, analysis of variance (ANOVA) $P=0.01$. Dox, doxycycline. Inset shows average distribution of haematopoietic colony types. c.f.u.-GEMM, c.f.u. granulocyte-erythroid-myeloid-megakaryocytes; c.f.u.-G/M/GM, c.f.u. granulocytes/ macrophages/granulocyte-macrophages; c.f.u.-E, c.f.u. erythroid. Bar graphs represent average \pm s.e.m. Pictures show representative colonies: c.f.u.-GEMM (top), c.f.u.-G/M/GM (middle), c.f.u.-E (bottom). Scale bar, $200 \mu \mathrm{mm}$. $* P<0.05$, $* * P<0.01$. 

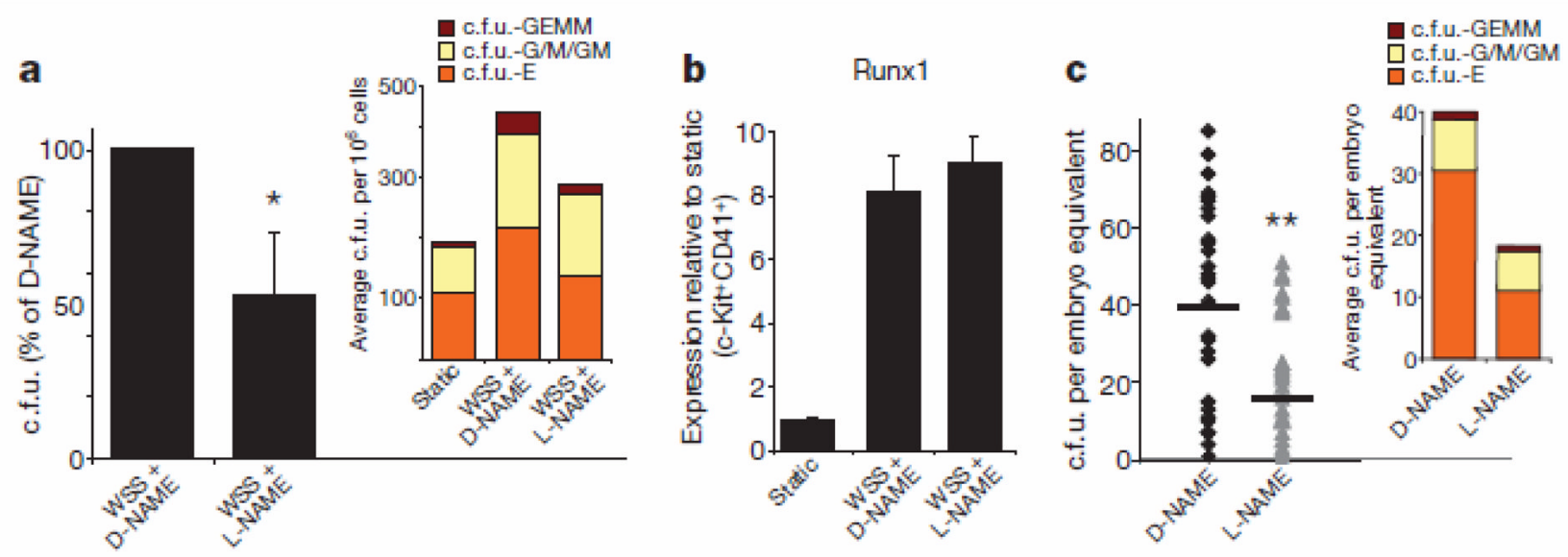

Figure 2. Nitric oxide production regulates the expansion of haematopoietic progenitors a, Methylcellulose haematopoietic c.f.u. assay. Pharmacological inhibition of nitric oxide synthesis with L-NAME reduces by $50 \%$ the WSS-mediated increase in haematopoietic c.f.u.; $n=3, P=0.04$. Inset shows the average distribution of colony types. $\mathbf{b}$, L-NAME does not affect WSS-mediated Runx1 upregulation; $n=3$. c, In vivo c.f.u. assay. Exposure of developing embryos to L-NAME from E8.5 to E10.5 leads to a reduction in haematopoietic progenitors in the AGM region. D-NAME, $n=30$; L-NAME, $n=32 ; P=0.0008$. Inset shows average distribution of colonies per AGM. Bar graphs represent average \pm s.e.m. $* P<0.05, * * P<0.005$. 


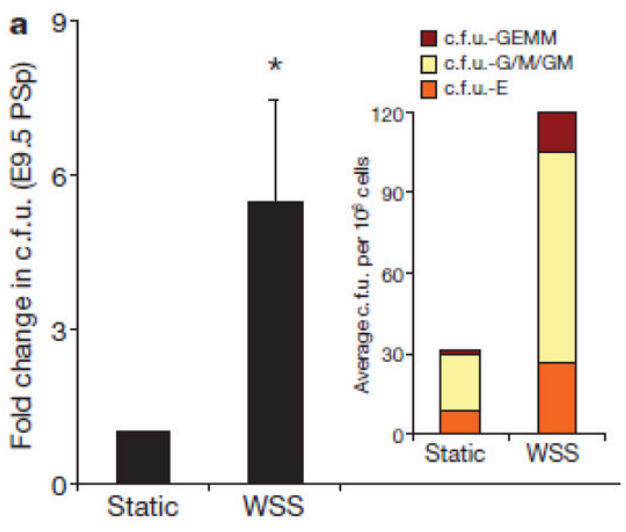

c
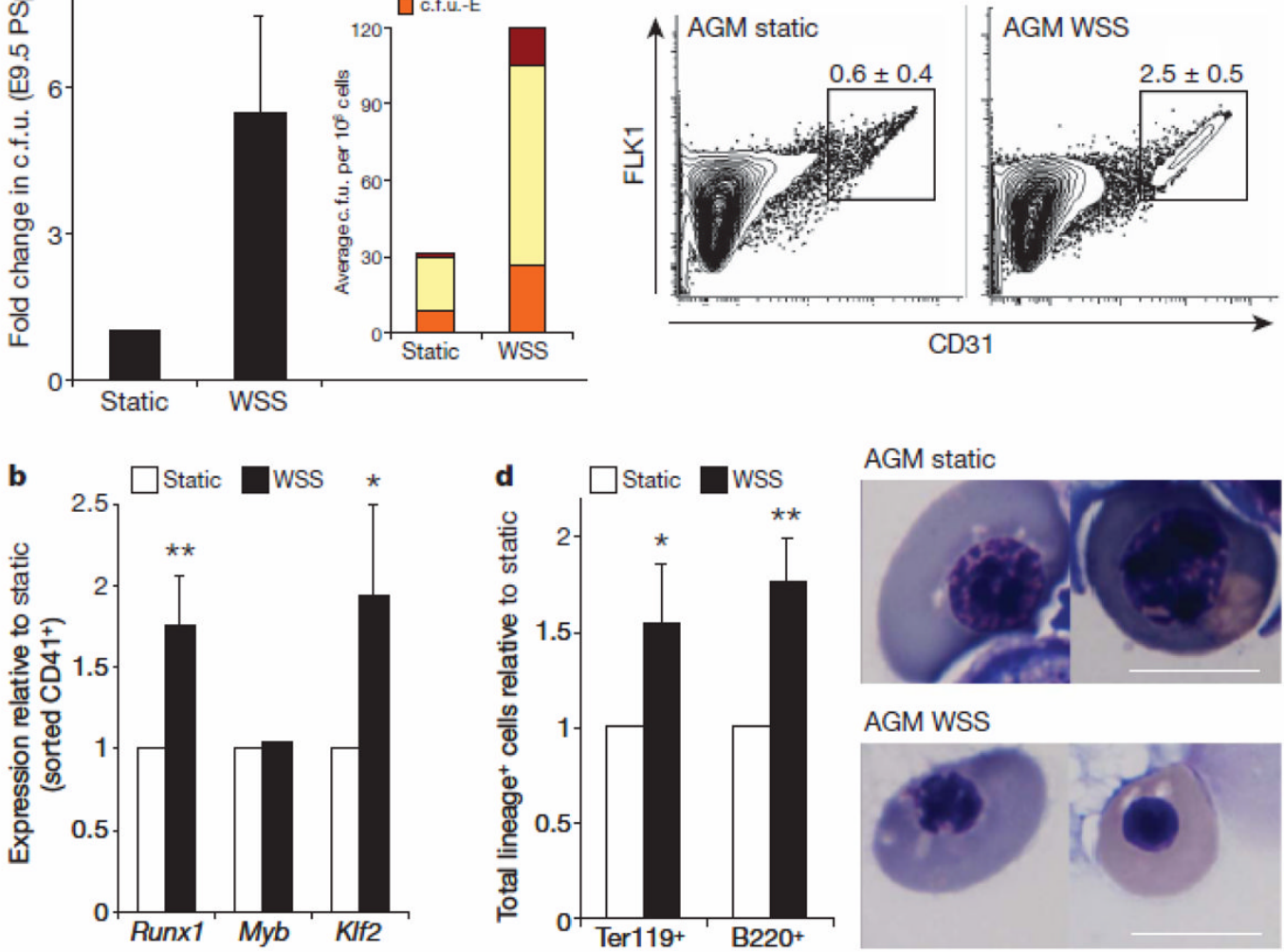

Figure 3. Shear stress induces haematopoiesis in PSp/AGM-embryo-derived cells

a, Methylcellulose haematopoietic c.f.u. assay. WSS increases the frequency of haematopoietic progenitors in two-dimensional primary PSp cultures from E9.5 embryos; $n=4(P=0.038)$;

bar graphs represent average \pm s.e.m. Inset shows average distribution of haematopoietic colony types. b, WSS induces upregulation of the haematopoietic markers Runxl $(P=0.01)$ and Klf2 $(P=0.05)$ in FACS-sorted AGM-derived CD41 $1^{+}$haematopoietic progenitors as documented by real-time PCR; $n=3$, bar graphs represent average \pm s.e.m. c, FACS analysis. WSS induces an increase in $\mathrm{CD} 31^{+}$cells in two-dimensional primary AGM cultures; $P=0.005$, $n=3$, average \pm s.d. d, WSS modulates the differentiation of AGM-derived haematopoietic progenitors as shown by an increase in absolute number of cells positive for the erythroid marker Ter119 $(P=0.02)$ and for the lymphoid marker B220 $(P=0.01) ; n=3$, bar graphs represent average \pm s.e.m. Shear stress induces maturation of erythroid precursors as documented by cell morphology in cytospins, which show pycnotic erythroblasts in WSStreated samples, and polychromatic erythroblasts in static cultures. Scale bar, $10 \mu \mathrm{m} . * P<0.05$, ** $P<0.01$. 

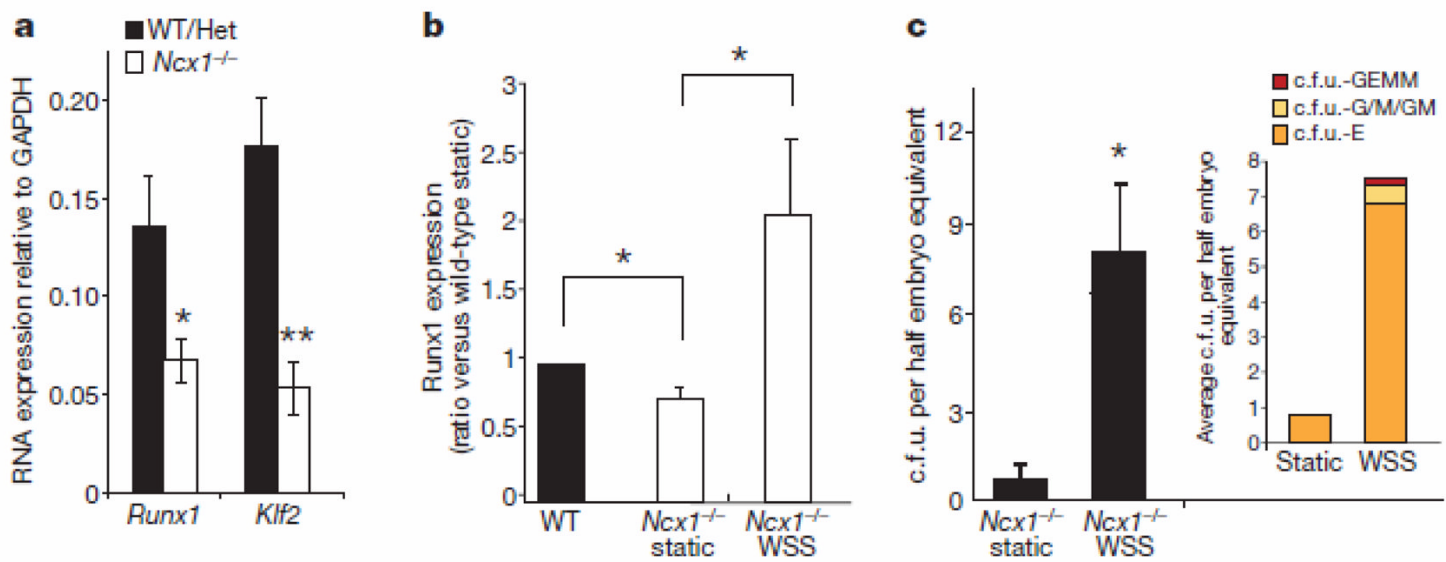

Figure 4. Runx1 expression and c.f.u. activity are shear-stress-dependent a, PSp isolated from E9.25 Ncx $1^{-l-}$ embryos show reduced gene expression levels of the haematopoietic markers Runxl $(P=0.02)$ and Klf2 $(P=0.003)$ when compared to matched wild-type (WT) or heterozygous (Het) littermate controls; $n=13 \mathrm{Ncxl}^{-/-}, n=15$ controls. b, Shear stress increases the expression of Runx1 in E9.25 Ncxl ${ }^{-1-}$ PSp cultures to levels comparable to the one observed in littermate controls; real-time quantitative PCR, $n=4$, ANOVA, $P=0.03$. Post-hoc multiple comparisons are two-tailed $t$-test with $P<0.02$. $c$, Shear stress induces c.f.u. activity in $N c x 1^{-/-}$PSp-derived cells. Average \pm s.e.m.; $P=0.01$. Inset shows average distribution of haematopoietic colony types at day 14 after replating; $n=6$. $* P<0.05, * * P<0.005$. 